\section{A case of vocal cord dysfunction with Takotsubo cardiomyopathy: is there a link?}

\author{
Xuan Ye, ${ }^{1}$ Sultana Aziza, ${ }^{2}$ Sean Gomes, ${ }^{3}$ \\ William Lancashire, ${ }^{4}$ Paul S. Thomas ${ }^{1,2}$ \\ 'Prince of Wales Clinical School, Faculty \\ of Medicine, University of New South \\ Wales; Departments of ${ }^{2}$ Respiratory \\ Medicine and ${ }^{3}$ Cardiology, Prince of \\ Wales Hospital, Randwick; ${ }^{4}$ Port \\ Macquarie Base Hospital, Port Macquarie, \\ NSW, Australia
}

\begin{abstract}
Vocal cord dysfunction (VCD) is an underrecognised cause of dyspnoea as it is seldom life threatening, and also difficult to diagnose. However, there have been rare accounts of VCD, as in the present case, which have led to haemodynamic instability. This patient with VCD episodically developed acute pulmonary oedema, bilateral pleural effusions and Takotsubo cardiomyopathy, treated effectively with tracheostomy. This presentation hints at the possible existence of a common pathophysiological mechanism, namely, forced inspiration against a closed airway or sympathetic overstimulation.
\end{abstract}

\section{Introduction}

Vocal cord dysfunction (VCD) is the inappropriate adduction of the vocal cords during respiration resulting in a functional obstruction of the upper airways. It is thought to be psychogenic in nature and rarely results in any significant systemic illness. Thus, VCD is typically managed conservatively with education and speech therapy although rare reports of refractory VCD have required surgical intervention. ${ }^{1}$ We report an unusual case of VCD associated with acute pulmonary oedema (AP0), severe recurrent bilateral pleural effusions (BPE) and Takotsubo cardiomyopathy (TCM), which was treated with permanent tracheostomy.

\section{Case Report}

A 73-year-old woman presented with acute shortness of breath accompanied by severe hypertension (220/110 mmHg), APO and BPE.
The symptoms occurred at rest and were associated with breathlessness and choking upon inspiration. At least two episodes in the past year had resulted in loss of consciousness with evidence of APO. The patient was otherwise asymptomatic in the months between attacks. Her past medical history included left bundle branch-block, obstructive sleep apnoea (OSA), pulmonary embolism, generalised anxiety disorder, gout and cervical cancer 30 years ago. She did not smoke or drink alcohol. Physical examination was unremarkable between attacks apart from mild obesity. Examination during these episodes revealed a profound dyspnoea, cyanosis and hypertension (systolic blood pressure $>200 \mathrm{mmHg}$ ) with oxygen saturation $<90 \%$. Grunting, dysphonia, fine basal crackles and prolonged expiration were also noted. Each attack lasted between 5 and 10 minutes and during severe attacks, the patient exhibited sympathetic features with profuse sweating, tremor and tachycardia. The patient's arterial blood gas revealed Type 2 respiratory failure. Initial cardiac evaluation revealed significant electrocardiogram (ECG) changes with mild lateral ST segment depression, T-wave inversion (V2-V3) and an elevated pro-BNP of $1996 \mathrm{pmol} / \mathrm{L}$. Secondary causes for the patient's hypertension were excluded with renal arteriography and ultrasonography, echocardiography, serial serum normetanephrines/5HIAA and a negative vasculitic screen and normal coronary arteries. Pulmonary embolism was excluded with computed tomography (CT) pulmonary angiography. Mild restriction was noted on spirometry. Bronchoscopy demonstrated paradoxical vocal cord movement, suggestive of VCD. The patient experienced a severe VCD attack with hypotension shortly after the procedure. At the same time a diagnosis of TCM was made on transthoracic echocardiogram (Figure 1), which resolved after 72 hours. This was supported by elevated serum normetanephrines $[1.75 \mathrm{nmol} / \mathrm{L}(<0.90 \mathrm{nmol} / \mathrm{L})]$. VCD was confirmed via flexible laryngoscopy on the basis of poor vocal cord abduction and false cord fasciculation with a posterior chink. Central causes of the patient's symptoms were excluded by cerebral magnetic resonance imaging (MRI) and single-photon emission computed tomography (SPECT) and a permanent tracheostomy performed to relieve her of respiratory distress as she failed to respond to speech therapy and psychological treatment. Twenty-two months post-discharge, the patient has remained attack-free but has symptoms of depression.

\section{Discussion}

This appears to be the first case of VCD with
Correspondence: Paul S. Thomas, Department of Respiratory Medicine, Prince of Wales Hospital, Randwick NSW 2031, Australia.

Fax: +61.2.9382.4627 - Tel. +61.2.9382 4620 .

Email: paul.thomas@unsw.edu.au

Key words: vocal cord dysfunction, takotsubo cardiomyopathy, tracheostomy; neurogenic pulmonary oedema, negative pressure pulmonary oedema.

Contributions: $\mathrm{XY}$, manuscript preparation, conception of ideas and literature review; SA, SG WL, clinical data collection, manuscript reviewing and cardiovascular imaging. PST, case report conception and manuscript preparation.

Conflict of interest: the authors report no conflicts of interest.

Received for publication: 2 June 2011.

Accepted for publication: 26 July 2011.

This work is licensed under a Creative Commons Attribution NonCommercial 3.0 License (CC BYNC 3.0).

CC Copyright X. Ye et al., 2011

Licensee PAGEPress, Italy

Chest Disease Reports 2011; 1:e8

doi:10.4081/cdr.2011.e8

APO, recurrent BPE and TCM. Although similar cardiopulmonary features have previously been reported, the instant case appears to be the first of intrinsic and non-traumatic origin. ${ }^{2,3}$ This manifestation of APO following acute upper airway obstruction suggests a common cause with two possible explanatory mechanisms: i) negative pressure pulmonary oedema (NPPO) or ii) neurogenic pulmonary oedema (NPO). Indeed, both phenomena are delayed in onset, typically occurring minutes to hours following the primary insult, which corresponds with the time course in this case. ${ }^{4,5}$

\section{Negative pressure pulmonary oedema hypothesis}

NPPO refers to the transudation of fluid into the pulmonary interstitium as a consequence of acute increases in negative intrathoracic pressure; the most common cause of this is forced inspiration against a closed larynx during laryngospasm..$^{5}$ It is proposed that this phenomenon is attributable to increases in pulmonary venous hydrostatic pressure resulting from a combination of increased right-sided systemic return (and therefore right ventricular output), impaired left-ventricular filling (due to distortion of cardiac geometry), and increased afterload (as verified by echocardiographical studies in healthy subjects). ${ }^{6}$ Further exacerbating this process is hypoxic vasoconstriction, which results from prolonged episodes of apnoea. 


\section{Neurogenic pulmonary oedema hypothesis}

NPO has been well documented in patients with severe central nervous system injury. ${ }^{4}$ In this case, the occurrence of NPO is likely to have resulted from either the direct effects of prolonged cerebral hypoxia or the indirect neurocirculatory effects of inspiration against a closed airway, both of which have been shown to trigger a significant sympathetic response. In the latter, studies on the neurophysiology of vigorous inspiratory efforts in healthy subjects and patients with OSA have demonstrated a marked increase in sympathetic tone following periods of apnoea as a rebound response to the intense vagal stimulation caused by large negative intrathoracic pressures. ${ }^{7,8}$ However, regardless of the precise mechanism, release of catecholamines may precipitate the transudation of fluid into the pulmonary interstitium via vasoconstriction and increased hydrostatic

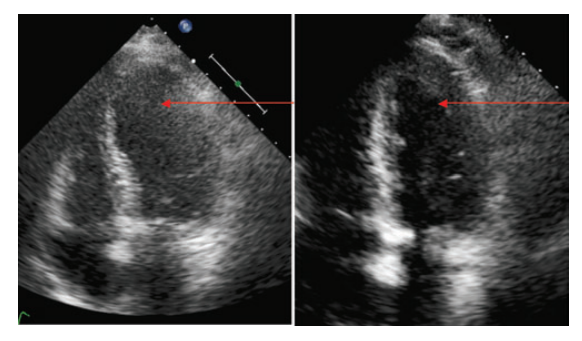

Figure 1. Takotsubo cardiomyopathy showing apical dilatation (arrow) with sparing of basal segments on transthoracic echocardiogram during systole (left), followed by complete recovery of the defect with normal left ventricular size (arrow) and function $48 \mathrm{~h}$ later (right). pressure. ${ }^{4}$ The elevated metanephrine level seen in our patient following her last episode supports this.

\section{Association with Takotsubo car- diomyopathy}

Either mechanism referred to above could explain the occurrence of APO in our case, but the coexistence of TCM strongly supports a neurogenic origin. TCM, also known as stress-induced cardiomyopathy, is a transient akinesis of the myocardium, which results in reversible left ventricular systolic impairment. The condition is classically associated with localised dilatation of the ventricular apex, and is thought to result from the deleterious effects of a catecholamine surge. In our patient, this occurred within 24 hours of her last (and most prolonged) attack. Other case reports have also documented the occurrence of TCM following acute severe upper airway obstruction by a giant glottic polyp and near drowning, but contain limited discussion of the potential mechanisms. ${ }^{2,3}$

\section{Conclusions}

VCD is commonly regarded as a benign selflimiting condition, but occasionally may be associated with cardiopulmonary sequelae. Extreme cases of VCD may precipitate haemodynamic instability, although the pathophysiological mechanisms remain speculative. While VCD may be an unusual cause of acute cardiac dysfunction, patients suffering from acute upper airway obstruction should be closely monitored for the occurrence of cardiopulmonary complications.

\section{References}

1. Goldstein R, Bright J, Jones SM, Niven RM. Severe vocal cord dysfunction resistant to all current therapeutic interventions. Respir Med 2007;101:857-8.

2. Citro R, Previtali M, Bossone E. Tako-tsubo cardiomyopathy and drowning syndrome: is there a link? Chest 2008;134:469.

3. Akiko S, Kengo Y, Manabu H, Yu 0. Takotsubo-like left ventricular dysfunction associated with impacted giant vocal cord polyp. J Jap Soc Intensive Care Med 2005;12:213-7.

4. Šedy J, Zicha J, Kunes J, et al. Mechanisms of neurogenic pulmonary edema development. Physiol Res 2008;57: 499-506.

5. Lowery JE, Myers LL. Florid negative pressure pulmonary edema. The Internet Journal of Otorhinolaryngology. 2008 Volume 7 Number 2.

6. Koshino Y, Villarragga HR, Orban M, et al. Changes in left and right ventricular mechanics during the Mueller maneuver in healthy adults: a possible mechanism for abnormal cardiac function in patients with obstructive sleep apnea. Circ Cardiovasc Imaging 2010;3:282-9.

7. Katragadda S, Xie A, Puleo D, et al. Neural mechanism of the pressor response to obstructive and nonobstructive apnea. J Appl Physiol 1997;83:2048-54.

8. Morgan BJ, Denahan T, Ebert TJ. Neurocirculatory consequences of negative intrathoracic pressure vs. asphyxia during voluntary apnea. J Appl Physiol 1993;74:2969-75. 